\title{
Automated Detection of Non-Termination and NullPointerExceptions for Java Bytecode*
}

\author{
Marc Brockschmidt, Thomas Ströder, Carsten Otto, and Jürgen Giesl \\ LuFG Informatik 2, RWTH Aachen University, Germany
}

\begin{abstract}
Recently, we developed an approach for automated termination proofs of Java Bytecode (JBC), which is based on constructing and analyzing termination graphs. These graphs represent all possible program executions in a finite way. In this paper, we show that this approach can also be used to detect non-termination or NullPointerExceptions. Our approach automatically generates witnesses, i.e., calling the program with these witness arguments indeed leads to non-termination resp. to a NullPointerException. Thus, we never obtain "false positives". We implemented our results in the termination prover AProVE and provide experimental evidence for the power of our approach.
\end{abstract}

\section{Introduction}

To use program verification in the software development process, one is not only interested in proving the validity of desired program properties, but also in providing a witness (i.e., a counterexample) if the property is violated.

Our approach is based on our earlier work to prove termination of JBC [4, $6,17]$. Here, a JBC program is first automatically transformed to a termination graph by symbolic evaluation. Afterwards, a term rewrite system is generated from the termination graph and existing techniques from term rewriting are used to prove termination of the rewrite system. As shown in the annual International Termination Competition, ${ }^{1}$ our corresponding tool AProVE is currently among the most powerful ones for automated termination proofs of Java programs.

Termination graphs finitely represent all runs through a program for a certain set of input values. Similar graphs were used for many kinds of program analysis (e.g., to improve the efficiency of software verification [7], or to ensure termination of program optimization [22]). In this paper, we show that termination graphs can also be used to detect non-termination and NullPointerExceptions.

In Sect. 2, we recapitulate termination graphs. In contrast to $[4,6,17]$, we also handle programs with arrays and we present an algorithm to merge abstract states in a termination graph which is needed in order to keep termination graphs finite. In Sect. 3 we show how to automatically generate witness states (i.e., suitable inputs to the program) which result in errors like NullPointerExceptions. Sect. 4 presents our approach to detect non-termination. Here, we use an SMT

\footnotetext{
* Supported by the DFG grant GI 274/5-3, the G.I.F. grant 966-116.6, and the DFG Research Training Group 1298 (AlgoSyn).

${ }^{1}$ See http://www.termination-portal.org/wiki/Termination_Competition
} 
solver to find different forms of non-terminating loops and the technique of Sect. 3 is applied to generate appropriate witness states.

Concerning the detection of NullPointerExceptions, most existing techniques try to prove absence of such exceptions (e.g., [15, 23]), whereas our approach tries to prove existence of NullPointerExceptions and provides counterexamples which indeed lead to such exceptions. So in contrast to bug finding techniques like $[2,9]$, our approach does not yield "false positives".

Methods to detect non-termination automatically have for example been studied for term rewriting (e.g., [11, 19]) and logic programming (e.g., [18]). We are only aware of two existing tools for automated non-termination analysis of Java: The tool Julia transforms JBC programs into constraint logic programs, which are then analyzed for non-termination [20]. The tool Invel [24] investigates non-termination of Java programs based on a combination of theorem proving and invariant generation using the KeY [3] system. In contrast to Julia and to our approach, Invel only has limited support for programs operating on the heap. Moreover, neither Julia nor Invel return witnesses for non-termination. In Sect. 5 we compare the implementation of our approach in the tool AProVE with Julia and Invel and show that our approach indeed leads to the most powerful automated non-termination analyzer for Java so far.

Moreover, [14] presents a method for non-termination proofs of C programs. In contrast to our approach, [14] can deal with non-terminating recursion and integer overflows. On the other hand, [14] cannot detect non-periodic non-termination (where there is no fixed sequence of program positions that is repeated infinitely many times), whereas this is no problem for our approach, cf. Sect. 4.2.

There also exist tools for testing $C$ programs in a randomized way, which can detect candidates for potential non-termination bugs (e.g., [13, 21]). However, they do not provide a proof for non-termination and may return "false positives".

\section{Termination Graphs}

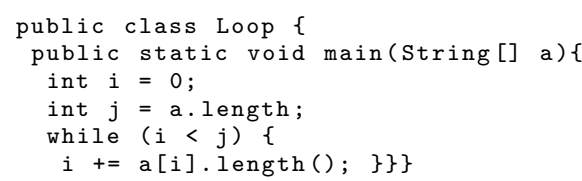

Fig. 1. Java Program

We illustrate our approach by the main method of the Java program in Fig. 1. The main method is the entry point when starting a program. Its only argument is an array of String objects corresponding to the arguments specified on the command line. To avoid dealing with all syntactic constructs of Java, we analyze JBC instead. JBC [16] is an assembly-like

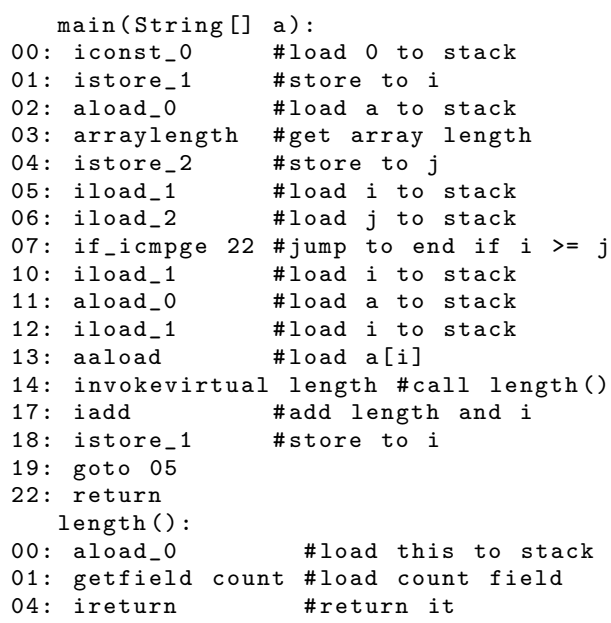

Fig. 2. JBC Program 
object-oriented language designed as intermediate format for the execution of Java. The corresponding JBC for our example, obtained automatically by the standard javac compiler, is shown in Fig. 2 and will be explained in Sect. 2.2.

The method main increments $i$ by the length of the $i$-th input string until $i$ exceeds the number $j$ of input arguments. It combines two typical problems:

(a) The accesses to a.length and $\mathrm{a}[\mathrm{i}]$.length () are not guarded by appropriate checks to ensure memory safety. Thus, if a or a [i] are null, the method ends with a NullPointerException. While this cannot happen when the method is used as an entry point for the program, another method could for instance contain String [] $b=\{$ null $\}$; Loop.main(b).

(b) The method may not terminate, as the input arguments could contain the empty string. If $a[i]=" "$, then the counter $i$ is not increased, leading to looping non-termination, as the same program state is visited again and again. For instance, the call java Loop " " does not terminate.

We show how to automatically detect such problems and to synthesize appropriate witnesses in Sect. 3 and 4. Our approach is based on termination graphs that over-approximate all program executions. After introducing our notion of states in Sect. 2.1, we describe the construction of termination graphs in Sect. 2.2. Sect. 2.3 shows how to create "merged" states representing two given states.

\subsection{Abstract States}

Our approach is related to abstract interpretation [8], since the states in termination graphs are abstract, i.e., they represent a (possibly infinite) set of concrete system configurations of the program. We define the set of all states as STATES $=$ $(\text { PPOS } \times \text { LOCVAR } \times \text { OPSTACK })^{*} \times(\{\perp\} \cup$ REFS $) \times$ HEAP $\times$ ANNOTATIONS.

Consider the program from Fig. 1 . The initial state $A$ in Fig. 3 represents all system configurations entering the main method with arbitrary tree-shaped (and thus, acyclic) non-null arguments. A state consists of four parts: the call stack, exception information, the heap, and annotations for possible sharing effects.

The call stack consists of stack frames, where several

$00\left|\mathrm{a}: a_{1}\right| \varepsilon$ $a_{1}$ :String[] $i_{1} \quad i_{1}:[\geq 0]$

Fig. 3. State $A$ frames may occur due to method calls. For readability, we exclude recursive programs, but our results easily extend to the approach of [6] for recursion. We also disregard multithreading, reflection, static fields, static initialization of classes, and floats.

Each stack frame has three components. We write the frames of the call stack below each other and separate their components by "l". The first component of a frame is the program position, indicated by the number of the next instruction (00 in Fig. 3). The second component represents the local variables by a list of references to the heap, i.e., LOCVAR $=$ REFs* ${ }^{*}$. To avoid a special treatment of primitive values, we also represent them by references. In examples, we write the names of variables instead of their indices. Thus, "a: $a_{1}$ " means that the value of the 0 -th local variable a is the reference $a_{1}$ (i.e., $a_{1}$ is the address of an array object). Of course, different local variables can point to the same address. The third component is the operand stack that JBC instructions work on, where OPSTACK $=$ REFS". The empty stack is " $\varepsilon$ " and " $i_{6}, i_{4}$ " is a stack with $i_{6}$ on top. 
Information about thrown exceptions is represented in the second part of our states. If no exception is currently thrown, this part is $\perp$ (which we do not display in example states). Otherwise it is a reference to the exception object.

Below the call stack, information about the heap is given by a partial function from HEAP $=$ REFS $\rightarrow$ (INTEGERS $\cup$ UNKNOWN $\cup$ INSTANCES $\cup$ ARRAYS $\cup\{$ null $\}$ ) and by a set of annotations which specify possible sharing effects.

Our representation of integers abstracts from the different bounded types of integers in Java and considers arbitrary integer numbers instead (i.e., we do not handle overflows). To represent unknown integer values, we use possibly unbounded intervals, i.e., INTEGERS $=\{\{x \in \mathbb{Z} \mid a \leq x \leq b\} \mid a \in \mathbb{Z} \cup\{-\infty\}, b \in$ $\mathbb{Z} \cup\{\infty\}, a \leq b\}$. We abbreviate $(-\infty, \infty)$ by $\mathbb{Z}$ and intervals like $[0, \infty)$ by $[\geq 0]$. So " $i_{1}$ : $[\geq 0]$ " means that any non-negative integer can be at the address $i_{1}$.

Classnames contains the names of all classes and interfaces in the program. Types $=$ Classnames $\cup\{t[] \mid t \in$ Types $\}$ contains Classnames and all resulting array types. So a type $t[]$ can be generated from any type $t$ to describe arrays with entries of type $t .^{2}$ We call $t^{\prime}$ a subtype of $t$ iff $t^{\prime}=t$; or $t^{\prime}$ extends ${ }^{3}$ or implements a subtype of $t$; or $t^{\prime}=\hat{t}^{\prime}[], t=\hat{t}[]$, and $\hat{t}^{\prime}$ is a subtype of $\hat{t}$.

The values in UNKNOWN $=$ TYPES $\times\{?\}$ represent tree-shaped (and thus acyclic) objects and arrays where we have no information except the type. For example, for a class List with the field next of type List, " $o_{1}$ : List(?)" means that the object at address $o_{1}$ is null or of a subtype of List.

INSTANCES represent objects of some class. They are described by the values of their fields, i.e., Instances $=$ Classnames $\times($ FieldIDs $\rightarrow$ Refs $)$. For cases where field names are overloaded, the FIELDIDs also contain the respective class name to avoid ambiguities, but we usually do not display it in our examples. So " $o_{1}:$ List $\left(\right.$ next $\left.=o_{2}\right)$ " means that at the address $o_{1}$, there is a List object and the value of its field next is $o_{2}$. For all $(c l, f) \in$ Instances, the function $f$ is defined for all fields of the class $c l$ and all of its superclasses.

In contrast to our earlier papers $[4,6,17]$, in this paper we also show how to handle arrays. An array can be represented by an element from TYPES $\times$ REFS denoting the array's type and length (specified by a reference to an integer value). For instance, " $a_{1}$ : String[] $i_{1}$ " means that at the address $a_{1}$, there is a String array of length $i_{1}$. Alternatively, the array representation can also contain an additional list of references for the array entries. So " $a_{2}$ : String[] $i_{1}\left\{o_{1}, o_{2}\right\}$ " denotes that at the address $a_{2}$, we have a String array of length $i_{1}$, and its entries are $o_{1}$ and $o_{2}$ (displayed in the Java syntax " $\{\ldots\}$ " for arrays). Thus, ARRAYS $=($ TYPES $\times$ REFS $) \cup\left(\right.$ TYPES $\times$ REFS $\times$ REFS $\left.{ }^{*}\right)$.

In our representation, no sharing can occur unless explicitly stated. So an abstract state containing the references $o_{1}, o_{2}$ and not mentioning that they could be sharing, only represents concrete states where $o_{1}$ and the references reachable from $o_{1}$ are disjoint ${ }^{4}$ from $o_{2}$ and the references reachable from $o_{2}$.

\footnotetext{
${ }^{2}$ We do not consider arrays of primitives in this paper, but our approach can easily be extended to handle them, as we did in our implementation.

${ }^{3}$ For example, any type (implicitly) extends the type java.lang. Object.

${ }^{4}$ Disjointness is not required for references pointing to INTEGERs or to null.
} 
Moreover, then the objects at $o_{1}$ and $o_{2}$ must be tree-shaped (and thus acyclic).

Certain sharing effects are represented directly (e.g., " $o_{1}: \operatorname{List}\left(\right.$ next $\left.=o_{1}\right)$ " is a cyclic singleton list). Other sharing effects are represented by three kinds of annotations, which are only built for references $o$ where $h(o) \notin$ INTEGERS $\cup\{$ null $\}$ for the heap $h$. The first kind of annotation is called equality annotation and has the form " $o_{1}=$ ? $o_{2}$ ". Its meaning is that the addresses $o_{1}$ and $o_{2}$ could be equal. We only use such annotations if the value of at least one of $o_{1}$ and $o_{2}$ is UnKNown. Joinability annotations are the second kind of annotation. They express that two objects "may join" $\left(o_{1} \downarrow o_{2}\right)$. We say that a non-integer and non-null reference $o^{\prime}$ is a direct successor of $o$ in a state $s$ (denoted $o \rightarrow_{s} o^{\prime}$ ) iff the object at address $o$ has a field whose value is $o^{\prime}$ or if the array at address $o$ has $o^{\prime}$ as one of its entries. The meaning of " $O_{1} \downarrow O_{2}$ " is that there could be an $o$ with $o_{1} \rightarrow_{s}^{*} O \leftarrow_{s}^{+} O_{2}$ or $o_{1} \rightarrow_{s}^{+} o \leftarrow_{s}^{*} O_{2}$, i.e., $o$ is a common successor of the two references. However, $o_{1} \downarrow o_{2}$ does not imply $o_{1}=$ ? $o_{2}$. Finally, as the third type of annotations, we use cyclicity annotations "o!" to denote that the object at address $o$ is not necessarily tree-shaped (so in particular, it could be cyclic).

\subsection{Constructing Termination Graphs}

Starting from the initial state $A$, the termination graph in Fig. 4 is constructed by symbolic evaluation. In the first step, we have to evaluate iconst_0, i.e., we load the integer 0 on top of the operand stack. The second instruction istore_1 stores the value 0 on top of the operand stack in the first local variable i. ${ }^{5}$

After that, the value of the 0-th local variable a (the array in the input argument) is loaded on the operand stack and the instruction arraylength retrieves its (unknown) length $i_{1}$. That value is then stored in the second local variable $j$ using the instruction istore_2. This results in the state $B$ in Fig. 4. We connect $A$ and $B$ by a dotted arrow, indicating several evaluation steps (i.e., we omitted the states between $A$ and $B$ for space reasons in Fig. 4).

From $B$ on, we load the values of $i$ and $j$ on the operand stack and reach $C{ }^{6}$ The instruction if_icmpge branches depending on the relation of the two elements on top of the stack. However, based on the knowledge in $C$, we cannot determine whether $i>=j$ holds. Thus, we perform a case analysis (called integer refinement [4, Def. 1]), obtaining two new states $D$ and $E$. We label the refinement edges from $C$ to $D$ and $E$ (represented by dashed arrows) by the reference $i_{1}$ that was refined. In $D$, we assume that $i>=j$ holds. Hence, $i_{1}$ (corresponding to $j$ ) is $\leq 0$ and from $i_{1}:[\geq 0]$ in state $C$ we conclude that $i_{1}$ is 0 . We thus reach instruction 22 (return), where the program ends (denoted by $\square$ ).

In $E$, we consider the other case and replace $i_{1}$ by $i_{2}$, which only represents positive integers. We mark what relation holds in this case by labeling the evaluation edge from $E$ to its successor with $0<i_{2}$. In general, we always use a

\footnotetext{
${ }^{5}$ If we have a reference whose value is from a singleton interval like $[0,0]$ or null, we replace all its occurrences in states by 0 resp. by null. So in state $B$, we simply write "i:0". Such abbreviations will also be used in the labels of edges.

${ }^{6}$ The box around $C$ and the following states is dashed to indicate that these states will be removed from the termination graph later on.
} 


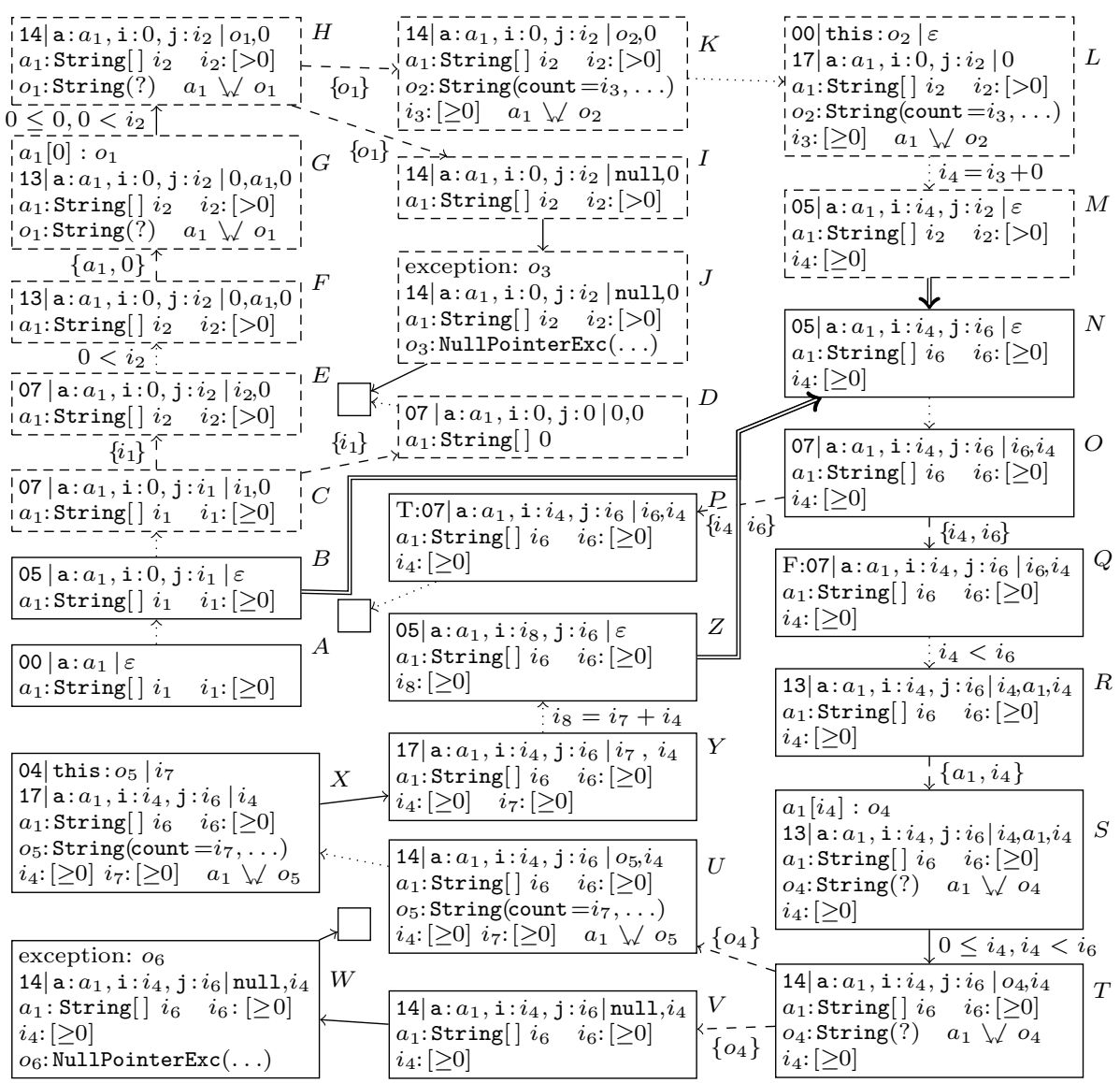

Fig. 4. Termination Graph

fresh reference name like $i_{2}$ when generating new values by a case analysis, to ensure single static assignments, which will be useful in the analysis later on. We continue with instruction 10 and load the values of $i, a$, and $i$ on the operand stack, obtaining state $F$. To evaluate aaload (i.e., to load the 0 -th element from the array $a_{1}$ on the operand stack), we add more information about $a_{1}$ at the index 0 and label the refinement edge from $F$ to $G$ accordingly. In $G$, we created some object $o_{1}$ for the 0 -th entry of the array $a_{1}$ and marked that $o_{1}$ is reachable from $a_{1}$ by adding the joinability annotation $a_{1} \downarrow o_{1} .^{7}$

Now evaluation of aaload moves $o_{1}$ to the operand stack in state $H$. Whenever an array access succeeds, we label the corresponding edge by the condition that the used index is $\geq 0$ and smaller than the length of the array.

In $H$, we need to invoke the method length () on the object $o_{1}$. However, we do not know whether $o_{1}$ is null (which would lead to a NullPointerException).

\footnotetext{
${ }^{7}$ If we had already retrieved another value $o^{\prime}$ from the array $a_{1}$, it would also have been annotated with $a_{1} \downarrow o^{\prime}$ and we would consequently add $o_{1} \downarrow o^{\prime}$ and $o_{1}={ }^{?} o^{\prime}$ when retrieving $o_{1}$, indicating that the two values may share or even be equal.
} 
Hence, we perform an instance refinement [4, Def. 5] and label the edges from $H$ to the new states $I$ and $K$ by the reference $o_{1}$ that is refined. In $I, o_{1}$ has the value null. In $K$, we replace the reference $o_{1}$ by $o_{2}$, pointing to a concrete String object with unknown field values. In Fig. 4, we only display the field count, containing the integer reference $i_{3}$. In this instance refinement, one uses the special semantics of the pre-defined String class to conclude that $i_{3}$ can only point to a non-negative integer, as count corresponds to the length of the string. In $I$, further evaluation results in a NullPointerException. A corresponding exception object $o_{3}$ is generated and the exception is represented in $J$. As no exception handler is defined, evaluation ends and the program terminates.

In $K$, calling length() succeeds. In $L$, a new stack frame is put on top of the call stack, where the implicit argument this is set to $o_{2}$. In the called method length (), we load $o_{2}$ on the operand stack and get the value $i_{3}$ of its field count. We then return from length (), add the returned value $i_{3}$ to 0 , and store the result in the variable $i$. Afterwards, we jump back to instruction 05. This is shown in state $M$ and the computation $i_{4}=i_{3}+0$ is noted on the evaluation edge.

But now $M$ is at the same program position as $B$. Continuing our symbolic evaluation would lead to an infinite tree, as we would always have to consider the case where the loop condition $i<j$ is still true. Instead, our goal is to obtain a finite termination graph. The solution is to automatically generate a new state $N$ which represents all concrete states that are represented by $B$ or $M$ (i.e., $N$ results from merging $B$ and $M$ ). Then we can insert instance edges from $B$ and $M$ to $N$ (displayed by double arrows) and continue the graph construction with $N$.

\subsection{Instantiating and Merging States}

To find differences between states and to merge states, we introduce state positions. Such a position describes a "path" through a state, starting with some local variable, operand stack entry, or the exception object and then continuing through fields of objects or entries of arrays. For the latter, we use the set ARRAYIDXS $=\{[j] \mid j \geq 0\}$ to describe the set of all possible array indices.

Definition 1 (State Positions SPos). Let $s=\left(\left\langle f r_{0}, \ldots, f r_{n}\right\rangle, e, h, a\right)$ be a state where each stack frame $f r_{i}$ has the form $\left(p p_{i}, l v_{i}, o s_{i}\right)$. Then $\operatorname{SPos}(s)$ is the smallest set containing all the following sequences $\pi$ :

- $\pi=\mathrm{LV}_{i, j}$ where $0 \leq i \leq n, l v_{i}=\left\langle o_{i, 0}, \ldots, o_{i, m_{i}}\right\rangle, 0 \leq j \leq m_{i}$. Then $\left.s\right|_{\pi}$ is $o_{i, j}$.

- $\pi=\mathrm{OS}_{i, j}$ where $0 \leq i \leq n$, os $s_{i}=\left\langle o_{i, 0}^{\prime}, \ldots, o_{i, k_{i}}^{\prime}\right\rangle, 0 \leq j \leq k_{i}$. Then $\left.s\right|_{\pi}$ is $o_{i, j}^{\prime}$.

- $\pi=\operatorname{ExC}$ if $e \neq \perp$. Then $\left.s\right|_{\pi}$ is $e$.

- $\pi=\pi^{\prime} v$ for some $v \in$ FIELDIDs and some $\pi^{\prime} \in \operatorname{SPOS}(s)$ where $h\left(\left.s\right|_{\pi^{\prime}}\right)=$ $(c l, f) \in$ Instances and where $f(v)$ is defined. Then $\left.s\right|_{\pi}$ is $f(v)$.

- $\pi=\pi^{\prime}$ LEN for some $\pi^{\prime} \in \operatorname{SPOS}(s)$ where $h\left(\left.s\right|_{\pi^{\prime}}\right)=(t, i) \in$ ARRAYs or $h\left(\left.s\right|_{\pi^{\prime}}\right)=(t, i, d) \in$ ARRAYs. Then $\left.s\right|_{\pi}$ is $i$.

- $\pi=\pi^{\prime}[j]$ for some $[j] \in \operatorname{ArRAYIDXs}$ and some $\pi^{\prime} \in \operatorname{SPOS}(s)$, where $h\left(\left.s\right|_{\pi^{\prime}}\right)=\left(t, i,\left\langle r_{0}, \ldots, r_{q}\right\rangle\right) \in$ ARRAYs and $0 \leq j \leq q$. Then $\left.s\right|_{\pi}$ is $r_{j}$.

For any position $\pi$, let $\bar{\pi}_{s}$ denote the maximal prefix of $\pi$ such that $\bar{\pi}_{s} \in \operatorname{SPOS}(s)$. We write $\bar{\pi}$ if $s$ is clear from the context. 
For example, in state $K$, the position $\pi=\mathrm{OS}_{0,0}$ count refers to the reference $i_{3}$, i.e., we have $\left.K\right|_{\pi}=i_{3}$ and for the position $\tau=\mathrm{LV}_{0,0}$ LEN, we have $\left.K\right|_{\tau}=i_{2}$. As the field count was introduced between $H$ and $K$ by an instance refinement, we have $\pi \notin \operatorname{SPOS}(H)$ and $\bar{\pi}_{H}=\operatorname{OS}_{0,0}$, where $\left.H\right|_{\bar{\pi}}=o_{1}$. We can now see that $B$ and $M$ only differ in the positions $\mathrm{LV}_{0,0} \mathrm{LEN}, \mathrm{LV}_{0,1}$, and $\mathrm{LV}_{0,2}$.

A state $s^{\prime}$ is an instance of another state $s$ (denoted $s^{\prime} \sqsubseteq s$ ) if both are at the same program position and if whenever there is a reference $\left.s^{\prime}\right|_{\pi}$, then either the values represented by $\left.s^{\prime}\right|_{\pi}$ in the heap of $s^{\prime}$ are a subset of the values represented by $\left.s\right|_{\pi}$ in the heap of $s$ or else, $\pi \notin \operatorname{SPOS}(s)$. Moreover, shared parts of the heap in $s^{\prime}$ must also be shared in $s$. As we only consider verified JBC programs, the fact that $s$ and $s^{\prime}$ are at the same program position implies that they have the same number of local variables and their operand stacks have the same size. For a formal definition of "instance", we refer to $[5,4] .{ }^{8}$

For example, $B$ is not an instance of $M$ since $h_{B}\left(\left.B\right|_{\mathrm{Lv}_{0,2}}\right)=[0, \infty) \nsubseteq[1, \infty)=$ $h_{M}\left(\left.M\right|_{\mathrm{LV}_{0,2}}\right)$ for the heaps $h_{B}$ and $h_{M}$ of $B$ and $M$. Similarly, $M \nsubseteq B$ because $h_{M}\left(\left.M\right|_{\mathrm{LV}_{0,1}}\right)=[0, \infty) \not \subset\{0\}=h_{B}\left(\left.B\right|_{\mathrm{LV}_{0,1}}\right)$. However, we can automatically synthesize a "merged" (or "widened") state $N$ with $B \sqsubseteq N$ and $M \sqsubseteq N$ by choosing the values for common positions $\pi$ in $B$ and $M$ to be the union of the values in $B$ and $M$, i.e., $h_{N}\left(\left.N\right|_{\pi}\right)=h_{B}\left(\left.B\right|_{\pi}\right) \cup h_{M}\left(\left.M\right|_{\pi}\right)$. Thus, we have $h_{N}\left(\left.N\right|_{\mathrm{LV}_{0,2}}\right)=[0, \infty) \cup[1, \infty)=[0, \infty)$ and $h_{N}\left(\left.N\right|_{\mathrm{LV}_{0,1}}\right)=\{0\} \cup[0, \infty)=[0, \infty)$.

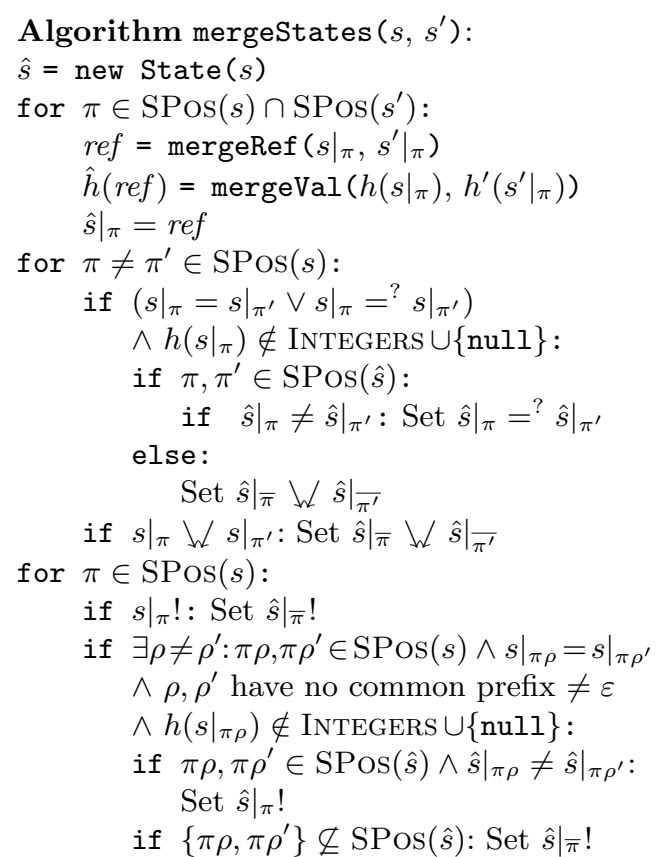

This merging algorithm is illustrated in Fig. 5. Here, $h, h^{\prime}, \hat{h}$ refer to the heaps of the states $s, s^{\prime}, \hat{s}$, respectively. With new State $(s)$, we create a fresh state at the same program position as $s$. The auxiliary function mergeRef is an injective mapping from a pair of references to a fresh reference name. The function mergeVal maps two heap values to the most precise value from our abstract domains that represents both input values. For example, mergeVal $([0,1],[10,15])$ is $[0,15]$, covering both input values, but also adding $[2,9]$ to the set of represented values. For values of the same type, e.g., String (count $\left.=i_{1}, \ldots\right)$ and String $\left(\right.$ count $\left.=i_{2}, \ldots\right)$, mergeVal returns a new object of same type with field values obtained by mergeRef, e.g., String (count $\left.=i_{3}, \ldots\right)$ where $i_{3}$ $=$ mergeRef $\left(i_{1}, i_{2}\right)$. When merging values of differing types or null, a

Fig. 5. Merging Algorithm

8 The "instance" definition from [4, Def. 3] can easily be extended to arrays, cf. [5]. 
value from UNKNOWN with the most precise common supertype is returned.

To handle sharing effects, in a second step, we check if there are "sharing" references at some positions $\pi$ and $\pi^{\prime}$ in $s$ or $s^{\prime}$ that do not share anymore in the merged state $\hat{s}$. Then we add the corresponding annotations to the maximal prefixes $\left.\hat{s}\right|_{\bar{\pi}}$ and $\left.\hat{s}\right|_{\pi^{\prime}}$. Furthermore, we check if there are non-tree shaped objects at some position $\pi$ in $s$ or $s^{\prime}$, i.e., if one can reach the same successor using different paths starting in position $\pi$. Then we add the annotation $\hat{s} \mid \bar{\pi}$ !

Theorem 2. Let $s, s^{\prime} \in \operatorname{StATES}$ and $\hat{s}=\operatorname{mergeStates}\left(s, s^{\prime}\right)$. Then $s \sqsubseteq \hat{s} \sqsupseteq s^{\prime} .^{9}$

In our example, we used the algorithm mergeStates to create the state $N$ and draw instance edges from $B$ and $M$ to $N$. Since the computation in $N$ also represents the states $C$ to $M$ (marked by dashed borders), we now remove them.

We continue symbolic evaluation in $N$, reaching state $O$, which is like $C$. In $C$, we refined our information to decide whether the condition $i \quad>=j$ of if_icmpge holds. However, now this case analysis cannot be expressed by simply refining the intervals from INTEGERS that correspond to the references $i_{6}$ and $i_{4}$ (i.e., a relation like $i_{4} \geq i_{6}$ is not expressible in our states). Instead, we again generate successors for both possible values of the condition $i>=j$, but do not change the actual information about our values. In the resulting states $P$ and $Q$, we mark the truth value of the condition $i>=j$ by "T" and "F". The refinement edges from $O$ to $P$ and $Q$ are marked by the references $i_{4}$ and $i_{6}$ that are refined. $P$ leads to a program end, while we continue the symbolic evaluation in $Q$. As before, we label the refinement edge from $Q$ to $R$ by $i_{4}<i_{6}$.

$R$ and $S$ are like $F$ and $G$. The refinement edge from $R$ to $S$ is labeled by $a_{1}$ and $i_{4}$ which were refined in order to evaluate aaload (note that since we only reach $R$ if $i_{4}<i_{6}$, the array access succeeds). As in $H$, we then perform an instance refinement to decide whether calling length() on the object $o_{4}$ succeeds, leading to $U$ and $V$. From $V$, we again reach a program end after a NullPointerException was thrown in $W$. From $U$, we reach $X$ by evaluating the call to length(). Between $X$ to $Y$, we return from length(). After that, we add the two non-negative integers $i_{7}$ and $i_{4}$, creating a non-negative integer $i_{8}$. The edge from $Y$ to $Z$ is labeled by the computation $i_{8}=i_{7}+i_{4}$.

$Z$ is again an instance of $N$. We can also use the algorithm mergeStates to determine whether one state is an instance of another: When merging $s, s^{\prime}$ to obtain a new state $\hat{s}$, one adapts mergeStates $\left(s, s^{\prime}\right)$ such that the algorithm terminates with failure whenever we widen a value of $s$ or add an annotation to $\hat{s}$ that did not exist in $s$ (e.g., when we add $\left.\hat{s}\right|_{\pi}=\left.{ }^{?} \hat{s}\right|_{\pi^{\prime}}$ and there is no $\left.s\right|_{\pi}=\left.{ }^{?} s\right|_{\pi^{\prime}}$ ). Then the algorithm terminates successfully iff $s^{\prime} \sqsubseteq s$ holds. After drawing the instance edge from $Z$ to $N$ (yielding a cycle in our graph), all leaves of the graph are program ends and thus the graph construction is finished.

We now define termination graphs formally. We extend our earlier definition from [4] slightly by labeling edges with information about the performed refinements and about the relations of integers. Let $\mathcal{R} e l \mathcal{O} p=\left\{i \circ i^{\prime} \mid i, i^{\prime} \in \operatorname{REFS}, \circ \in\right.$ $\{<, \leq,=, \neq, \geq,>\}\}$ denote the set of relations between two integer references such as $i_{4}<i_{6}$ and $\mathcal{A}$ rith $\mathcal{O} p=\left\{i=i^{\prime} \bowtie i^{\prime \prime} \mid i, i^{\prime}, i^{\prime \prime} \in \operatorname{REFS}, \bowtie \in\{+,-, *, /, \%\}\right\}$

\footnotetext{
${ }^{9}$ For all proofs, we refer to [5].
} 
denote the set of arithmetic computations such as $i_{8}=i_{7}+i_{4}$.

Termination graphs are constructed by repeatedly expanding those leaves that do not correspond to program ends. Whenever possible, we use symbolic evaluation $\stackrel{S y E v}{\longrightarrow}$. Here, $\stackrel{S y E v}{\longrightarrow}$ extends the usual evaluation relation for JBC such that it can also be applied to abstract states representing several concrete states. For a formal definition of $\stackrel{S y E v}{\longrightarrow}$, we refer to [4, Def. 6]. In the termination graph, the corresponding evaluation edges can be labeled by a set $C \subseteq \mathcal{A}$ rith $\mathcal{O} p \cup \mathcal{R}$ elO $p$ which corresponds to the arithmetic operations and (implicitly) checked relations in the evaluation. For example, when accessing the index $i$ of an array a succeeds, we have implicitly ensured $0 \leq i$ and $i<$ a.length and this is noted in $C$.

If symbolic evaluation is not possible, we refine the information for some references $R$ by case analysis and label the resulting refinement edges with $R$.

To obtain a finite graph, we create a more general state by merging whenever a program position is visited a second time in our symbolic evaluation and add appropriate instance edges to the graph. However, we require all cycles of the termination graph to contain at least one evaluation edge. By using an appropriate strategy for merging resp. widening states, we can automatically generate a finite termination graph for any program.

Definition 3 (Termination Graph). A graph $(V, E)$ with $V \subseteq$ States, $E \subseteq$ $V \times\left(\left(\{\right.\right.$ EVAL $\left.\} \times 2^{\mathcal{A} \text { rith } \mathcal{O} p \cup \mathcal{R} e l \mathcal{O} p}\right) \cup\left(\{\right.$ Refine $\left.\left.\} \times 2^{\mathrm{REFS}}\right) \cup\{\mathrm{INS}\}\right) \times V$ is a termination graph if every cycle contains at least one edge labeled with some $\mathrm{EvAL}_{C}$ and one of the following holds for each $s \in V$ :

- $s$ has just one outgoing edge $\left(s, \operatorname{EvAL}_{C}, s^{\prime}\right), s \stackrel{S y E v}{\longrightarrow} s^{\prime}$, and $C$ is the set of integer relations that are checked (resp. generated) in this step

- the outgoing edges of $s$ are $\left(s, \operatorname{REFINE}_{R}, s_{1}\right), \ldots,\left(s, \operatorname{REFINE}_{R}, s_{n}\right)$ and $\left\{s_{1}\right.$, $\left.\ldots, s_{n}\right\}$ is a refinement of $s$ on the references $R \subseteq$ REFS

- $s$ has just one outgoing edge ( $s$, INs, $\left.s^{\prime}\right)$ and $s \sqsubseteq s^{\prime}$

- $s$ has no outgoing edge and $s=(\varepsilon, e, h, a)$ (i.e., $s$ is a program end)

The soundness proofs for the transformation from JBC to termination graphs can be found in [4]. There, we show that if $c$ is a concrete state with $c \sqsubseteq s$ for some state $s$ in the termination graph, then the JBC evaluation of $c$ is represented in the termination graph. We refer to $[6,17]$ for methods to use termination graphs for termination proofs. In Sect. 3 and 4 we show how to use termination graphs to detect NullPointerExceptions and non-termination.

\section{Generating Witnesses for NullPointerExceptions}

In our example, an uncaught NullPointerException is thrown in the "error state" $W$, leading to a program end. Such violations of memory safety can be immediately detected from the termination graph. ${ }^{10}$

To report such a possible violation of memory safety to the user, we now show

${ }^{10}$ In C, memory safety means absence of (i) accesses to null, (ii) dangling pointers, and (iii) memory leaks [25]. In Java, the JVM ensures (ii) and (iii), and only NullPointerExceptions and ArrayIndexOutOfBoundsExceptions can destroy memory safety. 
how to automatically generate a witness (i.e., an assignment to the arguments of the program) that leads to the exception. Our termination graph allows us to generate such witnesses automatically. This technique for witness generation will also be used to construct witnesses for non-termination in Sect. 4 .

So our goal is to find a witness state $A^{\prime}$ for the initial state $A$ of the method main w.r.t. the "error state" $W$. This state $A^{\prime}$ describes a subset of arguments, all of which lead to an instance of $W$, i.e., to a NullPointerException.

Definition 4 (Witness State). Let $s, s^{\prime}, w \in$ States. The state $s^{\prime}$ is a witness state for $s$ w.r.t. $w$ iff $s^{\prime} \sqsubseteq s$ and $s^{\prime} \stackrel{S y E v_{*}}{\longrightarrow} w^{\prime}$ for some state $w^{\prime} \sqsubseteq w$.

To obtain a witness state $A^{\prime}$ for $A$ automatically, we start with the error state $W$ and traverse the edges of the termination graph backwards until we reach $A$. In general, let $s_{0}, s_{1}, \ldots, s_{n}=w$ be a path in the termination graph from the initial state $s_{0}$ to the error state $s_{n}$. Assuming that we already have a witness state $s_{i}^{\prime}$ for $s_{i}$ w.r.t. $w$, we show how to generate a witness state $s_{i-1}^{\prime}$ for $s_{i-1}$ w.r.t. $w$. To this end, we revert the changes done to the state $s_{i-1}$ when creating the state $s_{i}$ during the construction of the termination graph (i.e., we apply the rules for termination graph construction "backwards"). Of course, this generation of witness states can fail (in particular, this happens for error states that are not reachable from any concrete instantiation of the initial state $s_{0}$ ). So in this way, our technique for witness generation is also used as a check whether certain errors can really result from initial method calls.

In our example, the error state is $W$. Trivially, $W$ itself is a witness state for $W$ w.r.t. $W$. The only edge leading to $W$ is from $V$. Thus, we now generate a witness state $V^{\prime}$ for $V$ w.r.t. $W$. The edge from $V$ to $W$ represents the evaluation of the instruction invokevirtual that triggered the exception. Reversing this instruction is straightforward, as we only have to remove the exception object from $W$ again. Thus, $V$ is a witness state for $V$ w.r.t. $W$.

The only edge leading to $V$ is a refinement edge from $T$. As a refinement corresponds to a case analysis, the information in the target state is more precise. Hence, we can reuse the witness state for $V$, since $V$ is an instance of $T$. So $V$ is also a witness state for $T$ w.r.t. $W$.

$13\left|\mathrm{a}: a_{2}, \mathrm{i}: 0, \mathrm{j}: 1\right| 0, a_{2}, 0$

To reverse the edge between $T$ and $S$, we have to undo the instruction aaload. This is easy since $S$ contains the $a_{2}$ : String[ $] 1$ null $\}$

Fig. 6. State $R^{\prime}$ information that the entry at index $i_{4}$ in the array $a_{1}$ is $o_{4}$. Thus the witness state $S^{\prime}$ for $S$ w.r.t. $W$ is like $S$, but here $o_{4}$ 's value is not an unknown object, but null. Reversing the refinement between $S$ and $R$ is more complex. Note that not every state represented by $R$ leads to a NullPointerException. In $S$ we had noted the relation between the newly created reference $o_{4}$ and the original array $a_{1}$. In other words, in $S$ we know that $a_{1}\left[i_{4}\right]$ is $o_{4}$, where $o_{4}$ has the value null in the witness state $S^{\prime}$ for $S$. But in $R, o_{4}$ is missing. To solve this problem, in the witness state $R^{\prime}$ for $R$, we instantiate the abstract array $a_{1}$ by a concrete one that contains the entry null at the index $i_{4}$. We use a simple heuristic ${ }^{11}$ to choose a suitable

${ }^{11}$ Such heuristics cannot affect soundness, but just the power of our approach (choosing unsuitable values may prevent us from finding a witness for the initial state). 
length $i_{6}$ for this concrete array, which tries to find "minimal" values. Here, our heuristic chooses $a_{1}$ to be an array of length one (i.e., $i_{6}$ is chosen to be 1 ), which only contains the entry null (at the index 0 , i.e., $i_{4}$ is chosen to be 0 ). The resulting witness state $R^{\prime}$ for $R$ w.r.t. $W$ is displayed in Fig. 6 .

Reversing the evaluation steps between $R$ and $Q$ yields $0 \mid$ a: $a_{2} \mid \varepsilon$ a witness state $Q^{\prime}$ for $Q$ w.r.t. $W$. From $O$ to $Q$, we have a $\quad \begin{aligned} & a: \operatorname{string}[] 1\{\text { nul1 }\} \\ & a_{2} \text { St }\end{aligned}$ refinement edge and thus, $Q^{\prime}$ is also a witness for $O$.

Fig. 7. State $A^{\prime}$

The steps from $N$ to $O$ can also be reversed easily. In $N$, we use a heuristic to decide whether to follow the incoming edge from $Z$ or from $B$. Our heuristic chooses $B$ as it is more concrete than $Z$. From there, we continue our reversed evaluation until we reach a witness state $A^{\prime}$ for the initial state $A$ of the method w.r.t. $W$, cf. Fig. 7. So any instance of $A^{\prime}$ evaluates to an instance of $W$, i.e., it leads to a NullPointerException. If the main method is called directly (as the entry point of the program), then the JVM ensures that the input array does not contain null references. But if the main method is called from another method, then this violation of memory safety can indeed occur, cf. problem (a) in Sect. 2.

The following theorem summarizes our procedure to generate witness states. If there is an edge from a state $s_{1}$ to a state $s_{2}$ in the termination graph and we already have a witness state $s_{2}^{\prime}$ for $s_{2}$ w.r.t. $w$, then Thm. 5 shows how to obtain a witness state $s_{1}^{\prime}$ for $s_{1}$ w.r.t. $w$. Hence, by repeated application of this construction, we finally obtain a witness state for the initial state of the method w.r.t. $w$. If there is an evaluation edge from $s_{1}$ to $s_{2}$, then we first apply the reversed rules for symbolic evaluation on $s_{2}^{\prime}$. Afterwards, we instantiate the freshly appearing references (for example, those overwritten by the forward symbolic evaluation) such that $s_{1}^{\prime}$ is indeed an instance of $s_{1}$. If there is a refinement edge from $s_{1}$ to $s_{2}$, then the witness state $s_{1}^{\prime}$ is like $s_{2}^{\prime}$, but when reading from abstract arrays (such as between $R$ and $S$ ), we instantiate the array to a concrete one in $s_{1}^{\prime}$. If there is an instance edge from $s_{1}$ to $s_{2}$, then we intersect the states $s_{1}$ and $s_{2}^{\prime}$ to obtain a representation of those states that are instances of both $s_{1}$ and $s_{2}^{\prime}$.

Theorem 5 (Generating Witnesses). Let $\left(s_{1}, l, s_{2}\right)$ be an edge in the termination graph and let $s_{2}^{\prime}$ be a witness state for $s_{2}$ w.r.t. $w$. Let $s_{1}^{\prime} \in$ STATES with:

- if $l=\operatorname{EvAL}_{C}$, then $s_{1}^{\prime}$ is obtained from $s_{2}^{\prime}$ by applying the symbolic evaluation used between $s_{1}$ and $s_{2}$ backwards. In $s_{1}^{\prime}$, we instantiate freshly appearing variables such that $s_{1}^{\prime} \sqsubseteq s_{1}$ and $s_{1}^{\prime} \stackrel{\text { SyEv }}{\longrightarrow} s_{2}^{\prime}$ holds.

- if $l=\operatorname{REFINE}_{R}$, then $s_{1}^{\prime} \sqsubseteq s_{2}^{\prime}$.

- if $l=\mathrm{INS}$, then $s_{1}^{\prime}=s_{1} \cap s_{2}^{\prime}$ (for the definition of $\cap$, see [6, Def. 2]).

Then $s_{1}^{\prime}$ is a witness state for $s_{1}$ w.r.t. $w$.

\section{Proving Non-Termination}

Now we show how to prove non-termination automatically. Sect. 4.1 introduces a method to detect looping non-termination, i.e., infinite evaluations where the interesting references (that determine the termination behavior) are unchanged. Sect. 4.2 presents a method which can also detect non-looping non-termination. 


\subsection{Looping Non-Termination}

For each state, we define its interesting references that determine the control flow and hence, the termination behavior. Which references are interesting can be deduced from the termination graph, because whenever the (changing) value of a variable may influence the control flow, we perform a refinement. Hence, the references in the labels of refinement edges are "interesting" in the corresponding states. For example, the references $i_{4}$ and $i_{6}$ are interesting in the state $O$.

We propagate the information on interesting references backwards. For evaluation edges, those references that are interesting in the target state are also interesting in the source state. Thus, $i_{4}$ and $i_{6}$ are also interesting in $N$.

When drawing refinement or instance edges, references may be renamed. But if a reference at position $\pi$ is interesting in the target state of such an edge, the reference at $\pi$ is also interesting in the source state. So $i_{8}=\left.Z\right|_{\mathrm{Lv}_{0,1}}$ and $i_{6}=$ $\left.Z\right|_{\mathrm{Lv}_{0,2}}$ are interesting in $Z$, as $i_{4}=\left.N\right|_{\mathrm{Lv}_{0,1}}$ and $i_{6}=\left.N\right|_{\mathrm{Lv}_{0,2}}$ are interesting in $N$.

Furthermore, if an interesting reference $i$ of the target state was the result of some computation (i.e., the evaluation edge is labeled with $i=i^{\prime} \bowtie i^{\prime \prime}$ ), we mark $i^{\prime}$ and $i^{\prime \prime}$ as interesting in the source state. The edge from $Y$ to $Z$ has the label $i_{8}=i_{7}+i_{4}$. As $i_{8}$ is interesting in $Z, i_{7}$ and $i_{4}$ are interesting in $Y$.

Definition 6 (Interesting References). Let $G=(V, E)$ be a termination graph, and let $s, s^{\prime} \in V$ be some states. Then $I(s) \subseteq\left\{\left.s\right|_{\pi} \mid \pi \in \operatorname{SPOS}(s)\right\}$ is the set of interesting references of $s$, defined as the minimal set of references with

- if $\left(s, \operatorname{REFINE}_{R}, s^{\prime}\right) \in E$, then $R \subseteq I(s)$.

- if $\left(s, l, s^{\prime}\right) \in E$ with $l \in\left\{\operatorname{ReFine}_{R}\right.$, INS $\}$, then we have $\left\{\left.s\right|_{\pi} \mid \pi \in \operatorname{SPOS}(s) \cap\right.$ $\left.\operatorname{SPOs}\left(s^{\prime}\right),\left.s^{\prime}\right|_{\pi} \in I\left(s^{\prime}\right)\right\} \subseteq I(s)$.

- if $\left(s, \operatorname{Eval}_{C}, s^{\prime}\right) \in E$, then $I\left(s^{\prime}\right) \cap\left\{\left.s\right|_{\pi} \mid \pi \in \operatorname{SPos}(s)\right\} \subseteq I(s)$.

- if $\left(s, \operatorname{Eval}_{C}, s^{\prime}\right) \in E, i=i^{\prime} \bowtie i^{\prime \prime} \in C$ and $i \in I\left(s^{\prime}\right)$, then $\left\{i^{\prime}, i^{\prime \prime}\right\} \subseteq I(s)$.

Note that if there is an evaluation where the same program position is visited repeatedly, but the values of the interesting references do not change, then this evaluation will continue infinitely. We refer to this as looping non-termination.

To detect such non-terminating loops, we look at cycles $s=s_{0}, s_{1}, \ldots, s_{n-1}$, $s_{n}=s$ in the termination graph. Our goal is to find a state $v \sqsubseteq s$ such that when executing the loop, the values of the interesting references in $v$ do not change. More precisely, when executing the loop in $v$, one should reach a state $v^{\prime}$ with $v^{\prime} \sqsubseteq_{\Pi} v$. Here, $\Pi$ are the positions of interesting references in $s$ and $\sqsubseteq_{\Pi}$ is the "instance" relation restricted to positions with prefixes from $\Pi$, whereas the values at other positions are ignored. The following theorem proves that if one finds such a state $v$, then indeed the loop will be executed infinitely many times when starting the evaluation in a concrete instance of $v$.

Theorem 7 (Looping Non-Termination). Let s occur in a cycle of the termination graph. Let $\Pi=\left\{\pi \in \operatorname{SPOS}(s)|s|_{\pi} \in I(s)\right\}$ be the positions of interesting references in $s$. If there is a $v \sqsubseteq s$ where $v \stackrel{S y E v}{\longrightarrow}+v^{\prime}$ for some $v^{\prime} \sqsubseteq \Pi v$, then any concrete state that is an instance of $v$ starts an infinite JBC evaluation.

We now automate Thm. 7 by a technique consisting of four steps (the first 
three steps find suitable states $v$ automatically and the fourth step checks whether $v$ can be reached from the initial state of the method). Let $s=s_{0}, s_{1}, \ldots$, $s_{n-1}, s_{n}=s$ be a cycle in the termination graph such that there is an instance edge from $s_{n-1}$ to $s_{n}$. In Fig. $4, N, \ldots Z, N$ is such a cycle (i.e., here $s$ is $N$ ).

1. Find suitable values for interesting integer references. In the first step, we find out how to instantiate the interesting references of integer type in $v$. To this end, we convert the cycle $s=s_{0}, \ldots, s_{n}=s$ edge by edge to a formula $\varphi$ over the integers. Then every model of $\varphi$ indicates values for the interesting integer references that are not modified when executing the loop.

Essentially, $\varphi$ is a conjunction of all constraints that the edges are labeled with. More precisely, to compute $\varphi$, we process each edge $\left(s_{i}, l, s_{i+1}\right)$. If $l$ is REFINE $_{R}$, then we connect the variable names in $s_{i}$ and $s_{i+1}$ by adding the equations $\left.s_{i}\right|_{\pi}=\left.s_{i+1}\right|_{\pi}$ to $\varphi$ for all those positions $\pi$ where $\left.s_{i}\right|_{\pi}$ is in $R$ and points to an integer. Thus, for the edge from $O$ to $Q$, we add the trivial equations $i_{4}=i_{4} \wedge i_{6}=i_{6}$, as the references were not renamed in this refinement step.

If $l=\mathrm{EvAL}_{C}$, we add the constraints and computations from $C$ to the formula $\varphi .^{12}$ Thus, for the edge from $Q$ to $R$ we add the constraint $i_{4}<i_{6}$, for the edge from $S$ to $T$ we add $0 \leq i_{4} \wedge i_{4}<i_{6}$, and the edge from $Y$ to $Z$ yields $i_{8}=i_{7}+i_{4}$. If $l$ is INS, we again connect the reference names in $s_{i}$ and $s_{i+1}$ by adding the equations $\left.s_{i}\right|_{\pi}=\left.s_{i+1}\right|_{\pi}$ for all $\pi \in \operatorname{SPos}\left(s_{i+1}\right)$ that point to integers. Thus, for the edge from $Z$ to $N$, we get $i_{6}=i_{6} \wedge i_{8}=i_{4}$. So for the cycle $N, \ldots, Z, N, \varphi$ is $i_{4}<i_{6} \wedge 0 \leq i_{4} \wedge i_{8}=i_{7}+i_{4} \wedge i_{8}=i_{4}$ (where tautologies have been removed).

To find values for the integer references that are not modified in the loop, we now try to synthesize a model of $\varphi$. In our example, a standard SMT solver easily proves satisfiability and returns a model like $i_{4}=0, i_{6}=1, i_{7}=0, i_{8}=0$.

2. Guess suitable values for interesting non-integer references. We want to find a state $v \sqsubseteq s$ such that executing the loop does not change the values of interesting references in $v$. We have determined the values of the interesting integer references in $v$ (i.e., $i_{4}$ is 0 and $i_{6}$ is 1 in our example). It remains to determine suitable values for the other interesting references (i.e., for $a_{1}$ in our example)

$05\left|\mathrm{a}: a_{3}, \mathrm{i}: 0, j: 1\right| \varepsilon \quad$ To this end, we use the following heuristic. We instantiate $a_{3}:$ String[] 1 the integer references in $s_{n-1}$ according to the model found Fig. 8. State $Z^{\prime}$ for $\varphi$, yielding a state $s_{n-1}^{\prime} \sqsubseteq s_{n-1}$. So in our example (where $s_{n}=s$ is $N$ and $s_{n-1}$ is $Z$ ), we instantiate $i_{6}$ and $i_{8}$ in $Z$ by 1 resp. 0 , resulting in the state $Z^{\prime}$ in Fig. 8 (i.e., here $s_{n-1}^{\prime}$ is $Z^{\prime}$ ).

Afterwards, we traverse the path from $s_{n-1}$ backwards to $s_{0}$ and use the technique of witness generation from Sect. 3 to generate a witness $v$ for $s_{0}$ w.r.t. $s_{n-1}^{\prime}$

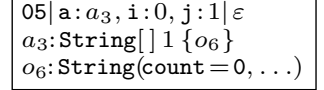

Fig. 9. State $N^{\prime}$ (i.e., $v \sqsubseteq s_{0}$ such that $v \stackrel{S y E v}{\longrightarrow}+v^{\prime}$ for some $\left.v^{\prime} \sqsubseteq s_{n-1}^{\prime}\right)$. In our example, ${ }^{13}$ the

$\overline{12}$ Remember that we use a single static assignment technique. Thus, we do not have to perform renamings to avoid name clashes.

${ }^{13}$ During the witness generation, one again uses the model of $\varphi$ for intermediate integer references. So when reversing the iadd evaluation between $Y$ and $Z$, we choose 0 as value for the newly appearing reference $i_{7}$. 
witness generation results in the state $N^{\prime}$ in Fig. 9. Note that the witness generation technique automatically "guessed" a suitable instantiation for the array (i.e., it was instantiated by a 1-element array containing just the empty string). Indeed, $N^{\prime} \sqsubseteq N$ and $N^{\prime} \stackrel{S y E v}{\longrightarrow}+v^{\prime}$ for an instance $v^{\prime}$ of $Z^{\prime}$ (i.e., in our example $s_{0}=s$ is $N$ and $v$ is $N^{\prime}$ ). Here, $v^{\prime}$ is like $Z^{\prime}$, but instead of " $a_{3}$ : String[] 1", we have " $a_{3}$ : String[ $] 1\left\{o_{6}\right\}$ " and " $o_{6}$ : String $($ count $=0, \ldots)$ ". Thus, $v^{\prime}=N^{\prime}$.

3. Check whether the guessed values for non-integer references do not change in the loop. While our construction ensures that the interesting integer references remain unchanged when executing the loop, this is not ensured for the interesting non-integer references. Hence, in the third step, we now have to check whether $v^{\prime} \sqsubseteq_{\Pi} v$ holds, where $\Pi$ are the positions of interesting references in $s$.

To this end, we adapt our algorithm mergeStates $\left(v, v^{\prime}\right)$ such that it terminates with failure whenever we widen a value of $v$ or add an annotation that did not exist in $v$ at a position with a prefix from $\Pi$. Then the algorithm terminates successfully iff $v^{\prime} \sqsubseteq_{\Pi} v$. In our example where $v=v^{\prime}=N$, we clearly have $v^{\prime} \sqsubseteq_{\Pi}$ $v$. Hence by Thm. 7, any instance of $v$ (i.e., of $N^{\prime}$ ) starts an infinite execution.

4. Check whether the non-terminating loop can be reached from the initial state. In the fourth step, we finally check whether $N^{\prime}$ can be reached from the initial state of the method. Hence, we again use the witness generation technique from Sect. 3 to create a witness state for $A$ w.r.t. $N^{\prime}$. This witness state has the stack frame "00 $\left|\mathrm{a}: a_{3}\right| \varepsilon$ " where $a_{3}$ is a 1-element array containing just the empty string. In other words, we automatically synthesized the counterexample to termination indicated in problem (b) of Sect. 2.

\subsection{Non-Looping Non-Termination}

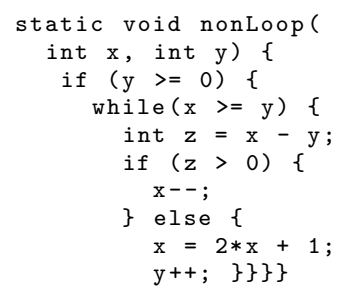

A loop can also be non-terminating if the values of interesting references are modified in its body. We now present a method to find such non-looping forms of nontermination. In contrast to the technique of Sect. 4.1, this method is restricted to loops that have no sub-loops and whose termination behavior only depends on integer arithmetic (i.e., the interesting references in all states of Fig. 10. nonLoop $(x, y)$ the loop may only refer to integers). Then we can construct a formula that represents the loop condition and the computation on each path through the loop. If we can prove that no variable assignment that satisfies the loop condition violates it in the next loop iteration, then we can conclude non-termination under the condition that the loop condition is satisfiable.

The method nonLoop in Fig. 11 does not terminate if $\mathrm{x} \geq \mathrm{y} \geq 0$. For example, if $\mathrm{x}=2, \mathrm{y}=1$ at the beginning of the loop, then after one iteration we have $\mathrm{x}=1, \mathrm{y}=1$. In the next iterations, we obtain $\mathrm{x}=3, \mathrm{y}=2 ; \mathrm{x}=2, \mathrm{y}=2$; and $\mathrm{x}=5, \mathrm{y}=3$, etc. So this non-termination is non-looping and even non-periodic (since there is no fixed sequence of program positions that is repeated infinitely many times). Thus, non-termination cannot be proved by techniques like [14].

Consider the termination graph, which is shown in a simplified version in 
Fig. 11. A node in a cycle with a predecessor outside of the cycle is called a loop head node. In Fig. 11, $A$ is such a node. We consider all paths $p_{1}, \ldots, p_{n}$ from the loop head node back to itself (without traversing the loop head node in between), i.e., $p_{1}=A, \ldots, B, A$ and $p_{2}=A, \ldots, C, A$. Here, $p_{1}$ corresponds to the case where $\mathrm{x} \geq \mathrm{y}$ and $\mathrm{z}=$ $\mathrm{x}-\mathrm{y}>0$, whereas $p_{2}$ handles the case where $\mathrm{x} \geq \mathrm{y}$ and $\mathrm{z}=\mathrm{x}-\mathrm{y} \leq 0$. For each path $p_{j}$, we generate a loop condition formula $\varphi_{j}$ (expressing the condition for entering this path) and a loop body formula $\psi_{j}$ (expressing how the values of the interesting references are changed in this path).

The formulas $\varphi_{j}$ and $\psi_{j}$ are generated as in Fig. 11. Graph for nonLoop Step 1 of Sect. 4.1, where we add relations from $\mathcal{R} e l \mathcal{O} p$ to $\varphi_{j}$ and constraints from $\mathcal{A}$ rith $\mathcal{O} p$ to $\psi_{j}$. In our example, $\varphi_{1}$ is $i_{1} \geq i_{2} \wedge i_{3}>0$ and $\varphi_{2}$ is $i_{1} \geq i_{2} \wedge i_{3} \leq 0$. Moreover, $\psi_{1}$ is $i_{3}=i_{1}-i_{2} \wedge i_{4}=i_{1}-1$ and $\psi_{2}$ is $i_{3}=i_{1}-i_{2} \wedge i_{5}=2 \cdot i_{1} \wedge i_{6}=$ $i_{5}+1 \wedge i_{7}=i_{2}+1$. To connect these formulas, we use a labeling function $\ell^{k}$ where for any formula $\xi, \ell^{k}(\xi)$ results from $\xi$ by labeling all variables with ${ }^{k}$. We use the labels ${ }^{1}, \ldots,{ }^{n}$ for the paths through the loop and the label ${ }^{r}$ for the resulting variables (in the second run, leaving the loop). We construct the formula

$$
\rho\left(p_{1}, \ldots, p_{n}\right)=\underbrace{\mu}_{\text {invariants }} \wedge \underbrace{\left(\bigvee_{j=1}^{n}\left(\ell^{j}\left(\varphi_{j}\right) \wedge \ell^{j}\left(\psi_{j}\right) \wedge \iota_{j}\right)\right)}_{\text {first run through the loop }} \wedge \underbrace{\left(\bigwedge_{j=1}^{n}\left(\neg \ell^{r}\left(\varphi_{j}\right) \wedge \ell^{r}\left(\psi_{j}\right)\right)\right)}_{\text {second run, leaving the loop }}
$$

Here, $\mu$ is a set of invariants that are known in the loop head node. So as we know " $i_{2}:[\geq 0]$ " in state $A, \mu$ is $i_{2} \geq 0$ for our example. The formula $\iota_{j}$ connects the variables labeled with ${ }^{j}$ to the unlabeled variables in $\mu$ and to the variables labeled with ${ }^{r}$ in the formulas for the second iteration. So for every integer reference $i$ in the loop head node, $\iota_{j}$ contains $i=i^{j}$. Moreover, if $i$ is an integer reference at position $\pi$ in the loop head node $s$ and $i^{\prime}$ is at position $\pi$ in the predecessor $s^{\prime}$ of $s$ (where there is an instance edge from $s^{\prime}$ to $s$ ), then $\iota_{j}$ contains $i^{\prime j}=i^{r}$. For our example, $\iota_{1}$ is $i_{1}=i_{1}^{1} \wedge i_{2}=i_{2}^{1} \wedge i_{4}^{1}=i_{1}^{r} \wedge i_{2}^{1}=i_{2}^{r}$.

Intuitively, satisfiability of the first two parts of $\rho\left(p_{1}, \ldots, p_{n}\right)$ corresponds to one successful run through the loop. The third part encodes that none of the loop conditions holds in the next run. Here, we do not only consider the negated conditions $\neg \ell^{r}\left(\varphi_{j}\right)$, but we also need $\ell^{r}\left(\psi_{j}\right)$, as $\varphi_{j}$ can contain variables computed in the loop body. For example in nonLoop, $\ell^{r}\left(\varphi_{1}\right)$ contains $i_{3}^{r}>0$. But to determine how $i_{3}^{r}$ results from the "input arguments" $i_{1}^{r}, i_{2}^{r}$, one needs $\ell^{r}\left(\psi_{1}\right)$ which contains $i_{3}^{r}=i_{1}^{r}-i_{2}^{r}$. If an SMT solver proves unsatisfiability of $\rho\left(p_{1}, \ldots, p_{n}\right)$, we know that whenever a variable assignment satisfies a loop condition, then after one execution of the loop body, a loop condition is satisfied again (i.e., the loop runs forever). Note that we generalized the notion of "loop conditions", as we discover the conditions by symbolic evaluation of the loop. Consequently, we can also handle loop control constructs like break or continue.

So unsatisfiability of $\rho\left(p_{1}, \ldots, p_{n}\right)$ implies that the loop is non-terminating, provided that the loop condition can be satisfied at all. To check this, we use an SMT solver to find a model for $\sigma\left(p_{1}, \ldots, p_{n}\right)=\mu \wedge\left(\bigvee_{j=1}^{n}\left(\ell^{j}\left(\varphi_{j}\right) \wedge \ell^{j}\left(\psi_{j}\right) \wedge \iota_{j}\right)\right)$. 
Theorem 8 (Non-Looping Non-Termination). Let $s$ be a loop head node in a termination graph where $I(s)$ only point to integer values and let $p_{1}, \ldots, p_{n}$ be all paths from $s$ back to $s$. Let $\rho\left(p_{1}, \ldots, p_{n}\right)$ be unsatisfiable and let $\sigma\left(p_{1}, \ldots, p_{n}\right)$ be satisfiable by some model $M$ (i.e., $M$ is an assignment of integer references to concrete integers). Let $c \sqsubseteq s$ be a concrete state where every integer reference in $c$ has been assigned the value given in $M$. Then $c$ starts an infinite JBC evaluation.

From the model $M$ of $\sigma\left(p_{1}, \ldots, p_{n}\right)$, we obtain an instance $v$ of the loop head node where we replace unknown integers by the values in $M$. Then the technique from Sect. 3 can generate a witness for the initial state of the method w.r.t. $v$. For our example, $i_{1}=i_{1}^{1}=i_{3}^{1}=1, i_{2}=i_{2}^{1}=i_{2}^{r}=i_{4}^{1}=i_{1}^{r}=0$ satisfies $\sigma\left(p_{1}, \ldots, p_{n}\right)$. From this, we obtain a witness for the initial state with $\mathrm{x}=1$ and $\mathrm{y}=0$, i.e., we automatically generate a non-terminating counterexample.

\section{Evaluation and Conclusion}

Based on termination graphs for Java Bytecode, we presented a technique to generate witnesses w.r.t. arbitrary error states. We then showed how to use this technique to prove the reachability of NullPointerExceptions or of nonterminating loops, which we detect by a novel SMT-based technique.

We implemented our new approach in the termination tool AProVE [12], using the SMT solver Z3 [10] and evaluated it on a collection of 325 examples. They consist of all 268 JBC programs from the Termination Problem Data Base that is used in the annual International Termination Competition, ${ }^{14}$ all 55 examples from [24] used to evaluate the Invel tool, and the two examples from this paper. For our evaluation, we compared the old version of AProVE (without support for non-termination), the new version AProVE-No containing the results of the present paper, and Julia [20]. We were not able to obtain a running version of Invel, and thus we only compared to the results of Invel reported in [24].

We used a time-out of 60 seconds for each example. "Yes" and "No" indicate how often termination (resp. non-termination) could be proved, "Fail" states how often the tool failed in less than 1 minute, "T" indicates how many examples

\begin{tabular}{|c|c|c|c|c|}
\hline & \multicolumn{2}{|c|}{ Invel Ex. } & \multicolumn{2}{|c|}{ Other Ex. } \\
\hline & \begin{tabular}{|l|l|l|}
$\mathbf{Y}$ & $\mathbf{N}$ & $\mathbf{I}$ \\
\end{tabular} & \begin{tabular}{|l|l|l|}
$\mathbf{F}$ & $\mathbf{T}$ & $\mathbf{R}$ \\
\end{tabular} & \begin{tabular}{l|l|l|}
$\mathbf{Y}$ & $\mathbf{N}$ & $\mathbf{F}$ \\
\end{tabular} & \begin{tabular}{|l|l}
$\mathbf{T}$ & $\mathbf{R}$ \\
\end{tabular} \\
\hline AProVE-No & \begin{tabular}{l|l|}
1 & 51
\end{tabular} & \begin{tabular}{l|l|l|}
0 & 3 & 5
\end{tabular} & \begin{tabular}{|l|l|l|}
204 & 30 & 12 \\
\end{tabular} & 241 \\
\hline AProVE & \begin{tabular}{|l|l|}
1 & 0
\end{tabular} & \begin{tabular}{|l|l|l|}
5 & 49 & 54 \\
\end{tabular} & \begin{tabular}{|l|l|l|}
204 & 0 & 27 \\
\end{tabular} & 391 \\
\hline Julia & \begin{tabular}{l|l|l}
1 & 0 & 5
\end{tabular} & 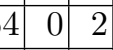 & \begin{tabular}{|l|l|l|}
166 & 22 & 82 \\
\end{tabular} & \\
\hline Invel & \begin{tabular}{l|l|l|l}
0 & 42 & 1
\end{tabular} & & & \\
\hline
\end{tabular}
led to a Time-out, and "R" gives the average Runtime in seconds for each example. The experiments clearly show the power of our contributions, since AProVE-No is the most powerful tool for automated non-termination proofs of Java resp. JBC programs. Moreover, the comparison between AProVE-No and AProVE indicates that the runtime for termination proofs did not increase due to the added non-termination techniques. To experiment with our implementation via a web interface and for details on the experiments, we refer to [1].

\section{References}

1. http://aprove.informatik.rwth-aachen.de/eval/JBC-Nonterm/.

\footnotetext{
${ }^{14}$ We removed a controversial example whose termination depends on integer overflows.
} 
2. N. Ayewah, D. Hovemeyer, J. D. Morgenthaler, J. Penix, and W. Pugh. Using static analysis to find bugs. IEEE Software, 25(5):22-29, 2008.

3. B. Beckert, R. Hähnle, and P. H. Schmitt. Verification of Object-Oriented Software: The KeY Approach. LNCS 4334. 2007.

4. M. Brockschmidt, C. Otto, C. von Essen, and J. Giesl. Termination graphs for Java Bytecode. In Verification, Induction, Termination Analysis, LNCS 6463, pages 1737, 2010. Extended version (with proofs) available at [1].

5. M. Brockschmidt, T. Ströder, C. Otto, and J. Giesl. Automated detection of nontermination and NullPointerExceptions for Java Bytecode. Report AIB 2011-19, RWTH Aachen, 2011. Available at [1] and at aib.informatik.rwth-aachen.de.

6. M. Brockschmidt, C. Otto, and J. Giesl. Modular termination proofs of recursive Java Bytecode programs by term rewriting. In Proc. RTA '11, LIPIcs 10, pages 155-170, 2011. Extended version (with proofs) available at [1].

7. R. Bubel, R. Hähnle, and R. Ji. Interleaving symbolic execution and partial evaluation. In Proc. FMCO '09, LNCS 6286, pages 247-277, 2010.

8. P. Cousot and R. Cousot. Abstract interpretation: A unified lattice model for static analysis of programs by construction or approximation of fixpoints. In Proc. POPL 'r7, pages 238-252. ACM Press, 1977.

9. C. Csallner, Y. Smaragdakis, and T. Xie. DSD-Crasher: A hybrid analysis tool for bug finding. ACM Trans. Softw. Eng. Methodol., 17:8:1-8:37, 2008.

10. L. de Moura and N. Bjørner. Z3: An efficient SMT solver. In Proc. TACAS '08, LNCS 4963, pages 337-340, 2008.

11. J. Giesl, R. Thiemann, P. Schneider-Kamp. Proving and disproving termination of higher-order functions. In Proc. FroCoS '05, LNAI 3717, pages 216-231, 2005.

12. J. Giesl, P. Schneider-Kamp, and R. Thiemann. AProVE 1.2: Automatic termination proofs in the dependency pair framework. In Proc. IJCAR '06, LNAI 4130, pages 281-286, 2006.

13. P. Godefroid, N. Klarlund, and K. Sen. DART: Directed automated random testing. In Proc. PLDI '05, pages 213-223. ACM Press, 2005.

14. A. Gupta, T. A. Henzinger, R. Majumdar, A. Rybalchenko, and R. Xu. Proving non-termination. In Proc. POPL '08, pages 147-158. ACM Press, 2008.

15. L. Hubert, T. Jensen, and D. Pichardie. Semantic foundations and inference of non-null annotations. In Proc. FMOODS '08, LNCS 5051, pages 132-149, 2008.

16. T. Lindholm and F. Yellin. Java Virtual Machine Specification. Prentice Hall, 1999.

17. C. Otto, M. Brockschmidt, C. von Essen, and J. Giesl. Automated termination analysis of Java Bytecode by term rewriting. In Proc. RTA '10, LIPIcs 6, pages 259-276, 2010. Extended version (with proofs) available at [1].

18. É. Payet and F. Mesnard. Nontermination inference of logic programs. $A C M$ Trans. Prog. Lang. Syst., 28:256-289, 2006.

19. É. Payet. Loop detection in term rewriting using the eliminating unfoldings. Theoretical Computer Science, 403:307-327, 2008.

20. É. Payet and F. Spoto. Experiments with non-termination analysis for Java Bytecode. In Proc. BYTECODE '09, ENTCS 5, pages 83-96, 2009.

21. K. Sen, D. Marinov, and G. Agha. CUTE: A concolic unit testing engine for C. In Proc. FSE '10, pages 263-272. ACM Press, 2005.

22. M. H. Sørensen and R. Glück. An algorithm of generalization in positive supercompilation. In Proc. ILPS '95, pages 465-479. MIT Press, 1995.

23. F. Spoto. Precise null-pointer analysis. Softw. Syst. Model., 10:219-252, 2011.

24. H. Velroyen and P. Rümmer. Non-termination checking for imperative programs. In Proc. TAP '08, LNCS 5051, pages 154-170, 2008.

25. H. Yang, O. Lee, J. Berdine, C. Calcagno, B. Cook, D. Distefano, P. O'Hearn. Scalable shape analysis for systems code. Proc. CAV'08, LNCS 5123, p. 385-398, 2008. 